\title{
ROTATION OF FOVEATED IMAGE IN THE WAVELET DOMAIN
}

\author{
Hang Yu Vu-Thanh Nguyen Ee-Chien Chang \\ School of Computing, \\ National University of Singapore \\ \{yuhang, nguyenvu, changec $\} @$ comp.nus.edu.sg
}

\begin{abstract}
An advantage of wavelet transform is its efficiency in representing natural images, that is, a natural image can be accurately represented by only a small number of retained wavelet coefficients. It is interesting to know whether some common image operations, e.g. rotation, can be performed very fast in wavelet domain. Preferably, the running time should depend only on the number of retained coefficients, not the size of the original image. However, it is not clear how this can be achieved. In this paper, we consider rotation, and images with a special structure: foveated images. Wavelet coefficients of a foveated image vanish outside an arrangement of circles. We exploit this structure to derive algorithms that accurately approximate rotation. The running time is $\Theta(m)$ where $m$ is the number of retained coefficients. Experiments show the accuracy of the approximation.
\end{abstract}

\section{INTRODUCTION}

Wavelet transform is known to be efficient in representing natural images, that is, a natural image can be accurately represented by only a small number of retained wavelet coefficients. Since the amount of data being processed is reduced, it brings forth the possibility of speeding up performance by operating only on the retained coefficients. Some works have exploited this characteristic for specific task, e.g. feature extraction [1]. Preferably, the running time should depend only on the number of retained coefficients. However, for some common image operations, e.g. rotation, it is not easy to achieve the speed-up. This is partly due to the "shift sensitive" characteristics of wavelet transform, i.e. a small shift in a signal generates unpredictable changes in its discrete wavelet transform coefficients. The shift insensitive complex wavelets could be employed to handle the translation [2] but it is not clear how they can be applied to handle slightly more complicated image operations like rotation and shearing. Also note that in general, even if the original image has many zero coefficients, the operated image could have few or none zero coefficients. Therefore, it is impossible to have both fast and exact algorithm.

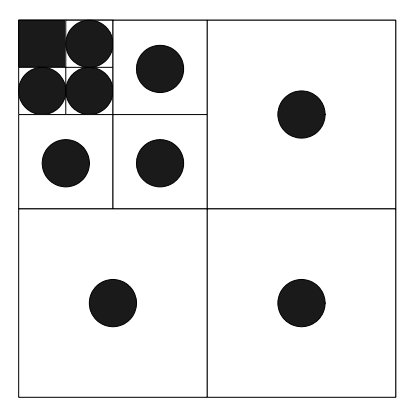

(a)

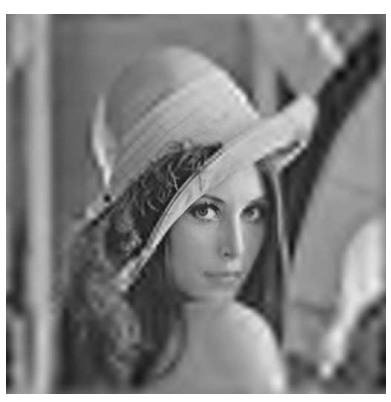

(b)
Fig. 1. (a) the mask when the fovea is at the center; (b) foveated image whose mask has radius of 30 and locates at center.

In this paper, we focus on rotation, and a particular type of image: foveated image. The resolution of a foveated image is highest at a point (fovea) and falling off away from the fovea. It is corresponding to our biological visual system, which has a space-variant nature where the resolution is high in the center (fovea) but decreasing towards the peripheral. As the biological visual system is highly effective, the space-variant nature of foveated image has inspired the design of many vision and imaging systems [3, 4]. An approximate foveated image can be obtained from the original image by multiplying the discrete wavelet transform (DWT) of the image by a predetermined 0-1 mask followed by the inverse discrete wavelet transform (IDWT) [4]. The mask indicates which coefficients are to be retained. Fig. 1(a) shows the mask when the fovea is at the center and Fig. 1(b) shows a foveated image $(512 \times 512)$ whose mask has radius of 30 and locates at the image's center. Observe that only a small number of coefficients are retained in the foveated image. More accurate approximation can be achieved by using a smoother mask, rather than the 0-1 mask [4].

We exploit the special structure of foveated image to derive fast algorithms that directly process foveated image in the wavelet domain. The running time is proportional to the 
number of coefficients retained. That is, we obtain a speedup from $\Theta\left(n^{2}\right)$ to $\Theta(m)$ where $n^{2}$ is the number of pixels, and $m$ is the number of coefficients retained. We propose two algorithms. The second algorithm is more accurate but the first algorithm is faster by a constant factor. Thus, theoretically, both algorithms run in $\Theta(m)$ time. However, it is instructive to present both algorithms.

\section{THE PROBLEM AND PROPOSED METHODS}

\subsection{The problem}

Consider a foveated image $I_{1}$ and its rotated version $I_{2}$. The input of our problem is $W_{1}$, the wavelet coefficients of $I_{1}$, and the output is $W_{2}$, an approximation of the wavelet coefficients of $I_{2}$.

A direct method requires three steps: (i) reconstructing the foveated image $I_{1}$ from $W_{1}$, i.e. applying IDWT on $W_{1}$, (ii) rotating the image $I_{1}$ to obtain $I_{2}$ and (iii) applying DWT on $I_{2}$ to get $W_{2}$.

Assuming that the image size is $n \times n$ pixels, each of the above steps requires a running time in the order of $\Theta\left(n^{2}\right)$, which is considerable when the image is large. This is especially so when higher order of interpolation is used during rotation. Recall that the $W_{1}$ can be represented by small number of coefficients. Let $m$ the number of coefficients retained during the foveation. In Fig. $1, m$ is the number of 1's in the mask. We want to design an algorithm that depends only on $m$.

Our algorithms are based on the observation that for a foveated image, the radius of the circles in the mask are the same across every level. Furthermore, the centers of the circles all correspond to the same location, which is the fovea, in the spatial domain. Consequently, we can focus the operation on the area around the fovea and significantly reduce the running time.

\subsection{Algorithm 1}

Consider the sub-bands in the wavelet transform. We call the $i$-th level LH, HL and HH high frequency sub-band $h_{i}$ (see Fig. 2(a)), and $l_{i}$ the LL sub-band of the $i$-th level. Thus $l_{3}$ can be reconstructed from $l_{1}, h_{1}$ and $h_{2}$. For a foveated image, its non-zero coefficients are concentrated in the arrangement of circles of radius $r$ shown in Fig. 1(a). We write $h_{i, r}$ as the coefficients retained in the sub-bands $h_{i}$. Thus, $h_{i, r}$ contains coefficients in 3 circles of radius $r$, where each circle corresponds to the LH, HL and HH subband.

Fig. 3 shows the steps of Algorithm 1 when the number of levels is 3. It is easy to generalize to any number of levels. In this figure, mask $\left(r_{0}\right)$ is an operation that applies a mask of radius $r_{0}$ in the sub-band. For example, after $l_{3}$ is applied a mask $(2 r)$, coefficients at a distant greater than
$2 r$ from the fovea will be removed (or equivalently, set to zeros). The operation "rotate" is the usual image rotation. We are not concerned with the interpolation method used during rotation. In our experiment, we employ bicubic interpolation.

At level 1, the inputs of Algorithm 1 are $l_{1}$ and $h_{1}$ of the foveated image. We apply IDWT on $l_{1}$ and $h_{1}$ to get $l_{2}$. Since the energy of $l_{2}$ is concentrated in the circle of radius $2 r$, to achieve speed-up, a circular mask of radius $2 r$ is applied to obtain $l_{2,2 r}$. Next, $l_{2,2 r}$ is rotated to produce a rotated $K_{2}$, follow by DWT to give the sub-bands $L_{1}$ and $H_{1}$. Similarly, the energy of each of these sub-bands is concentrated in a circle of radius $r$. Hence, we apply a mask of radius $r$ on $H_{1}$ to produce $H_{1, r}$. At level 2, similar process is repeated, except that the input is $l_{2, r}$. Note that masking is performed a few times in the above steps. Although masking reduces accuracy, it is necessary to make sure that the data size is small. After all, the values of the discarded coefficients are small. This recursive process is carried out until reaching the final level.

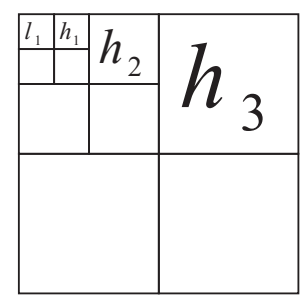

(a)

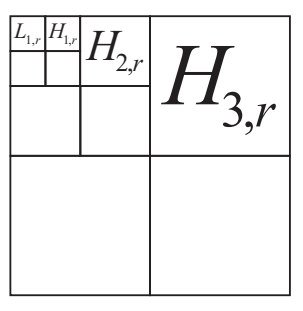

(b)

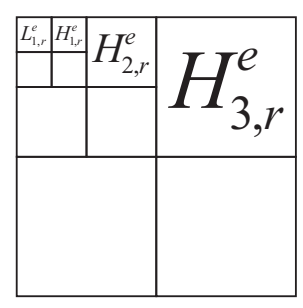

(c)
Fig. 2. Wavelet transforms of: (a) original foveated image; (b) foveated image rotated by Algorithm 1; (c) foveated image rotated by Algorithm 2.

Note that each of DWT, mask(), and rotations can be done in $\Theta\left(r^{2}\right)$ time and there are only $N$ levels. Thus the total time require is $\Theta\left(N r^{2}\right)$, which is $\Theta(m)$.

\subsection{Algorithm 2}

Algorithm 2 is a modified version of Algorithm 1. The main observation is the following. Let $K_{i, r}$ be the coefficients obtained by masking $K_{i}$ with the circle of radius $r$. Ideally, $K_{i, r}$ should be the same as $L_{i}$. However, this is not the case due to the combined effects of rotation and wavelet transformation. $L_{i}$ is more accurate because it is computed in 


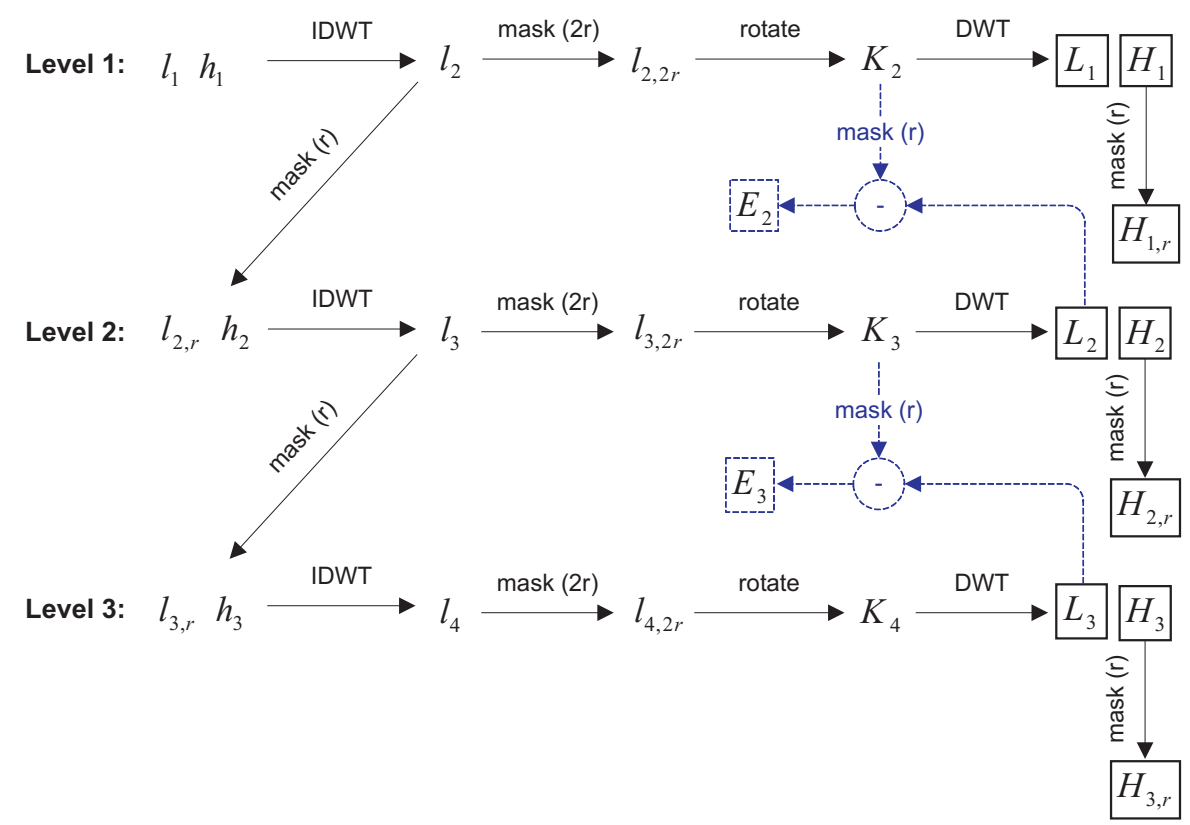

Fig. 3. Algorithm 1 to rotate foveated image directly in wavelet domain (dotted lines are for Algorithm 2).

the higher level. Therefore, if we retain $L_{i-1}, H_{i-1}$ which are calculated from $K_{i}$, in the final output, the LL sub-band at level $i$ will be the imprecise $K_{i}$.

In Algorithm 2, we introduce a step after $L_{i}$ is obtained. We first compute the error $E_{i}=L_{i}-K_{i, r}$. Next, DWT is applied on $E_{i}$. The wavelet transform of $E_{i}$ are then added to $L_{1}, H_{1}, H_{2}, \ldots, H_{i-1}$. To ensure $\Theta(m)$ computation, we approximate the DWT by restricting the coefficients in each sub-band to be in the circle of radius $r$.

\section{EXPERIMENTAL RESULTS}

We implement our proposed algorithms using several different images: "Lena" (512x512), "Mandrill" (512x512), "Cameraman" (256x256), "Peppers" (256x256) and "Barbara" (256x256). The performance of accuracy achieved is measured by Normalized Mean Square Error (NMSE). The wavelet filter used is the biorthogonal 7/11.

Fig. 4 shows the rotated image by different methods when the degree of rotation is 45 , and the radius of the mask is 15 . To clearly show the differences between images, we present their zoom-in versions whose focuses are around the fovea. We denote $J_{1}$ the image obtained by directly rotating the foveated image $I_{1}$ in the space domain, which is showed in Fig. 4(a). We denote $J_{2}$ the image obtained by a straightforward algorithm: applying DWT on $J_{1}$, mask on the wavelet coefficients and IDWT on the wavelet coefficients in the mask. The zoom-in of $J_{2}$ is shown in Fig. 4(b). We denote $A_{1}$ and $A_{2}$ the images obtained by our proposed Algorithm 1 and Algorithm 2, respectively. The zoom-in of
$A_{1}$ and $A_{2}$ are shown in Fig. 4(c) and Fig. 4(d). It is obvious that the image $A_{2}$ is more accurate than the image $A_{1}$ and comparable to $J_{1}$.

To compare the differences among $A_{1}, A_{2}, J_{1}$ and $J_{2}$, we use Normalized Mean Square Error (NMSE), which is the mean square error normalized by the energy of $J_{1}$. Fig. 5(a) shows the NMSE as the rotation degree increases and Fig. 5(b) shows the NMSE as the radius of the mask increases. Because of the rotation and wavelet transform, there may exist spike values on the boundaries of rotated images, which lead to inaccurate NMSE values. Therefore, we ignore the image boundary by comparing only the region within the inscribed circle of the image boundaries. The displayed NMSE values are the average results of 5 different images which we mentioned above.

We can see that the NMSE increases as the rotation degree increases, and decreases as the radius of the mask increases. It is because the larger the rotating degree is, the larger the number of non-zero coefficients are created in wavelet domain. It means that while the radius is unchanged, the number of non-zero coefficients being discarded (by our algorithms) increases and consequently NMSE increases. Similarly, when the radius increases, more number of coefficients are retained and we obtain more accurate results. The NMSE difference showed in Fig. 5 also confirms what we have visually concluded from Fig. 4 about the accuracy of the proposed algorithms. Note that the graph of $\left(J_{2}-J_{1}\right)$ indicates the best approximation ones can achieve. 


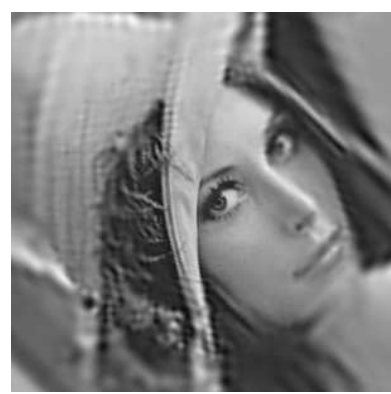

(a)

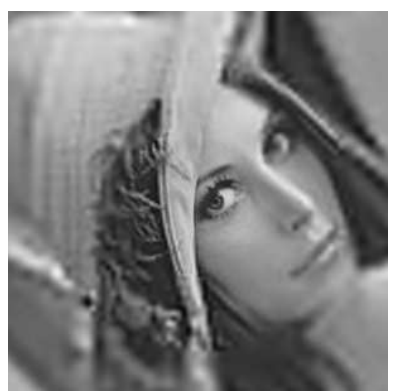

(b)

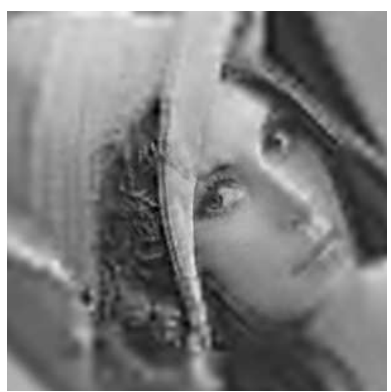

(c)

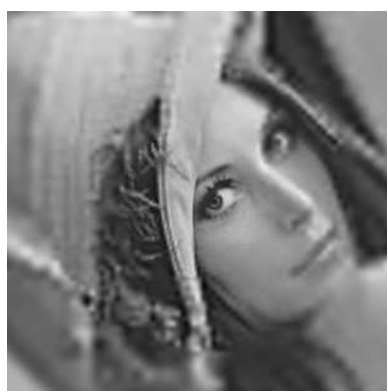

(d)

Fig. 4. Rotated images: (a) $J_{1}$ obtained by rotating the foveated image Fig. 1(b); (b) $J_{2}$ obtained by applying DWT on $J_{1}$ and IDWT on the coefficients in the mask; (c) $A_{1}$ obtained by Algorithm 1; (d) $A_{2}$ obtained by Algorithm 2.

\section{CONCLUSION}

Although wavelet transforms have been studied for more than a decade, there are few researches on direct and efficient manipulation in the wavelet domain. In this paper, we propose two algorithms that directly rotate foveated images in the wavelet-based compressed domain. The running time of our algorithms depends only on the number of retained coefficients, and this is considerably faster than the straightforward algorithm. While the proposed approximation algorithms are more efficient, experimental result shows that the approximation is also accurate. Here is a possible deployment of our algorithms: Suppose a method can efficiently extracts features of foveated images directly from its wavelet coefficients, and we want to extend it to rotated images. This extension can be done by first applying our algorithm, follows by the original efficient extraction method. In the future, it would be interesting to explore similar techniques for other operations.

\section{REFERENCES}

[1] Chang S.F., Smith J., "Extracting multi-dimensional signal features for content-based visual query," SPIE Visual Comm. and Image Processing, Taipei, May 1995.

[2] Fernandes F.C.A., van Spaendonck R.L.C., Burrus C.S. "A new framework for complex wavelet transforms," IEEE Trans. on Signal Processing, 51(7):1825-1837, July 2003.

[3] Burt B.J., "Smart sensing within a pyramid vision machine," Proc. of the IEEE, 76(8):1006-1015, 1988.

[4] Ee-Chien Chang, Stephane Mallat and Chee Yap, "Wavelet foveation," J. Applied and Computational Harmonic Analysis, 9(3):312-335, October 2000.

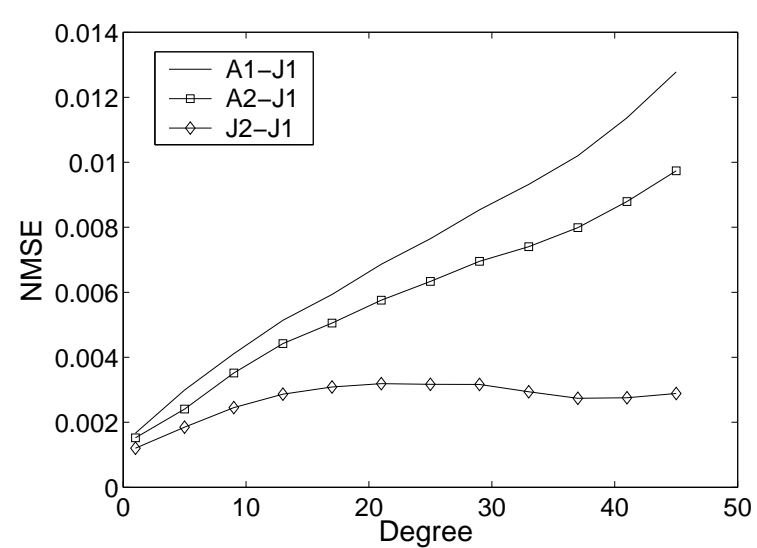

(a)

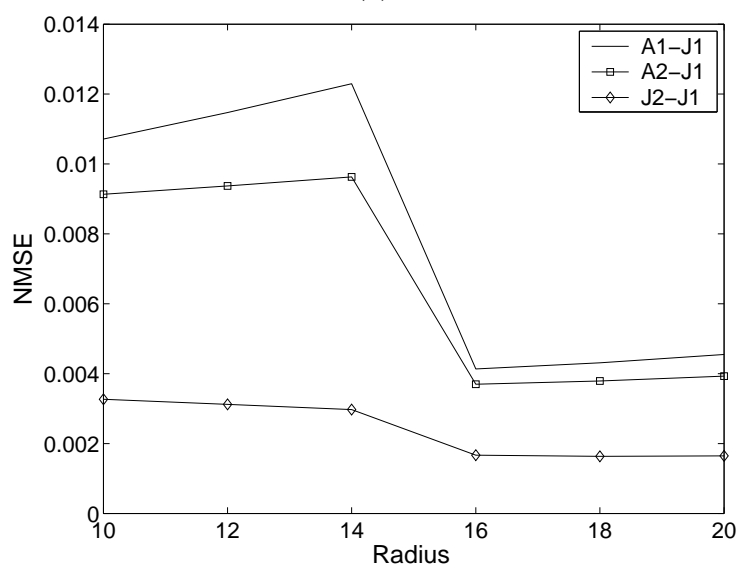

(b)

Fig. 5. Performance ratios: (a) NMSE as the rotating degree increases; (b) NMSE as the mask's radius increases. 